\title{
Tuning range and output power optimization of an external-cavity GaN diode laser at
} $455 \mathrm{~nm}$

\author{
Chi, Mingjun; Jensen, Ole Bjarlin; Petersen, Paul Michael
}

\section{Published in:}

Applied Optics

Link to article, DOI:

10.1364/AO.55.002263

Publication date:

2016

Document Version

Peer reviewed version

Link back to DTU Orbit

Citation (APA):

Chi, M., Jensen, O. B., \& Petersen, P. M. (2016). Tuning range and output power optimization of an externalcavity GaN diode laser at $455 \mathrm{~nm}$. Applied Optics, 55(9), 2263-2269. https://doi.org/10.1364/AO.55.002263

\section{General rights}

Copyright and moral rights for the publications made accessible in the public portal are retained by the authors and/or other copyright owners and it is a condition of accessing publications that users recognise and abide by the legal requirements associated with these rights.

- Users may download and print one copy of any publication from the public portal for the purpose of private study or research.

- You may not further distribute the material or use it for any profit-making activity or commercial gain

- You may freely distribute the URL identifying the publication in the public portal 


\title{
Tuning range and output power optimization of an external-cavity diode laser at $455 \mathrm{~nm}$
}

\author{
Minguun Chi, ${ }^{*}$ Ole Bjarlin Jensen, and Paul Michael Petersen
}

\author{
DTU Fotonik, Department of Photonics Engineering, Technical University of Denmark, Frederiksborgvej 399, P.O. Box 49, DK-4000 Roskilde, \\ Denmark \\ *Corresponding author: mchi@fotonik.dtu.dk
}

Received XX Month XXXX; revised XX Month, XXXX; accepted XX Month XXXX; posted XX Month XXXX (Doc. ID XXXXX); published XX Month XXXX

In this paper we discuss how different feedback gratings affect the tuning range and the output power of external feedback diode laser systems. A tunable high-power narrow-spectrum external-cavity diode laser system around $455 \mathrm{~nm}$ is investigated. The laser system is based on a high-power GaN diode laser in a Littrow external-cavity. Both a holographic diffraction grating and a ruled diffraction grating are used as feedback element in the external cavity. The output power, spectral bandwidth and tunable range of the external cavity diode laser system are measured and compared with the two gratings at different injected currents. When the holographic grating is used, the laser system can be tuned over a range of $1.4 \mathrm{~nm}$ with an output power around $530 \mathrm{~mW}$. When the ruled grating is used, the laser system can be tuned over a range of $6.0 \mathrm{~nm}$ with an output power around $80 \mathrm{~mW}$. The results can be used as a guide for selecting grating for external-cavity diode lasers for different requirements. C2015 Optical Society of America

OCIS codes: (140.5960) Semiconductor lasers; (140.2020) Diode lasers; (140.3600) Lasers, tunable; (140.4780) Optical resonators, (050.1950)

Diffraction gratings.

http://dx.doi.org/*****

\section{INTRODUCTION}

High-power, narrow-spectrum, tunable GaN lasers emitting from violet to blue range are attractive for many applications, such as highresolution spectroscopy, holographic data storage, laser cooling, and deep ultraviolet generation by second harmonic generation [1-5]. Since the first demonstration of room-temperature continuous-wave GaNbased violet diode lasers by Nakamura et alaround two decades ago [6], great progress has been achieved on the GaN based diode lasers. Today, high-power GaN diode lasers with continuous-wave output power of a few watts are commercially available $[7,8]$. But the spectral bandwidth of these high-power devices is around $1.0 \mathrm{~nm}$, and this broad emission bandwidth limits the usage of these devices in the applications mentioned above.

Different techniques have been applied to achieve narrow-linewidth, tunable GaN laser diodes, among them external-cavity feedback technique is one of the main approaches to improve the spectral quality of the GaN diode laser. An external-cavity diode laser system with output power up to $3.5 \mathrm{~mW}$ at $392 \mathrm{~nm}$ was demonstrated by Conroy et al. in 2000, with a $2.7 \mathrm{~nm}$ tunable range of the diode laser system [9]. Using an anti-reflection-coated InGaN device, a violet external-cavity diode laser system with a tunable range of $6.3 \mathrm{~nm}$ centered at $398 \mathrm{~nm}$ was achieved [10]. Around $410 \mathrm{~nm}$, an external-cavity diode laser system with an output power of $30 \mathrm{~mW}$ and a tuning range of $4 \mathrm{~nm}$ was demonstrated in 2003 [1]. Hult et al. showed a $450 \mathrm{~nm}$ extended-cavity diode laser with a maximum output power of $0.9 \mathrm{~mW}$, where the laser system had a mode-hop-free tuning range of $110 \mathrm{GHz}$ [11]. A narrowlinewidth external-cavity diode laser system was achieved around 405 $\mathrm{nm}$ in 2007, with an output power of around $80 \mathrm{~mW}$ [3]. Another 405 nm external-cavity diode laser was demonstrated by Lv et al. in 2013, with an output power of the laser around $1 \mathrm{~mW}$ and with a tuning range around $4.5 \mathrm{~nm}$ [12]. Recently, Ruhnke et al. reported an external-cavity diode laser at $445 \mathrm{~nm}$, where a maximum output power of $400 \mathrm{~mW}$ was obtained, and the tuning range was around $3 \mathrm{~nm}$ [13]. This externalcavity diode laser system was used as a pump source to generate deep violet radiation at $222.5 \mathrm{~nm}$ [5]. All the diode devices applied previously $[1,3,9-12]$ were narrow-stripe single lateral mode diode laser other than in Ref. [13], where a multi-lateral mode device was used.

In this paper, based on a high-power GaN diode laser in a Littrow external-cavity, we demonstrate a tunable high-power narrowspectrum diode laser system around $455 \mathrm{~nm}$. Two gratings, a holographic diffraction grating and a ruled diffraction grating are used in the external cavity. When the holographic grating is used, the laser system can be tuned over ranges of 3.1,2.8,2.4 and $1.4 \mathrm{~nm}$ with injected currents of $0.2,0.3,0.45$ and 0.6 A respectively. The output powers are around $85,200,360$ and $530 \mathrm{~mW}$ with the four injected currents. When the ruled grating is used, the laser system can be tuned over ranges of 6.0, 5.5 and $4.0 \mathrm{~nm}$ with injected currents of $0.3,0.6$ and $0.9 \mathrm{~A}$ respectively. The output powers are around 85,220 , and $340 \mathrm{~mW}$ with the three injected currents. The emission spectral bandwidth is less than $0.07 \mathrm{~nm}$ throughout the tuning ranges for both gratings. The results show, that the laser system has a higher efficiency and a narrower bandwidth when the holographic grating is used. However, the tunable range is much wider when the ruled grating is used. 


\section{EXPERIMENTAL SETUP}

The Littrow external-cavity configuration is one of the core externalcavity configurations applied to achieve tunable narrow-linewidth diode laser emission due to its compactness and simplicity compared with other external-cavity configurations such as the Littman externalcavity $[14,15]$. In the Littrow external-cavity geometry, the laser beam from one facet of a diode laser is collimated and incident on a diffraction grating mounted in Littrow configuration. The first-order diffracted beam from the grating is directed back into the diode laser, thus an external-cavity is formed between the grating and another facet of the laser. The zeroth-order diffracted beam or the beam from another facet of the laser is the output from the external cavity diode laser. Tuning of the external-cavity diode laser is achieved by rotating the diffraction grating. The Littrow external-cavity feedback technique has been applied to achieve tunable narrow-linewidth diode laser from the violet to the infrared spectral range $[9-13,16,17]$.

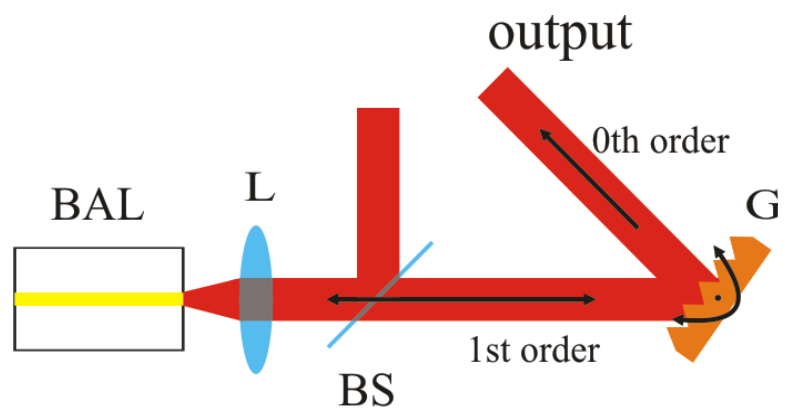

Fig. 1. Experimental set-up of the external-cavity blue diode laser system. BAL, broad-area diode laser; L, lens; BS, beam splitter; G, diffraction grating.

The external-cavity geometry employed in our experiment is depicted in Fig. 1. The GaN laser diode (DTR laser shop, M462) used in the experimentis a $2 \mathrm{~W}$ broad-area diode laser emitting around $455 \mathrm{~nm}$. The laser beam is TE-polarized, i.e., the polarization direction of the laser beam is parallel to the active region of the laser. The laser beam from the front facet is collimated by an aspherical lens of $4.0 \mathrm{~mm}$ focal length with a numerical aperture of 0.6 . The collimated beam is incident on a bulk diffraction grating, the grating is mounted in Littrow configuration and oriented with the lines in the grating parallel to the polarization direction of the laser beam. Two different bulk diffraction gratings are used in our experiment, one is a holographic diffraction grating with a groove density of 2400 lines/mm (Thorlabs, GH13-24U), while the other is a ruled diffraction grating which is ruled with 1800 lines $/ \mathrm{mm}$ and has a blaze wavelength of $500 \mathrm{~nm}$ (Thorlabs, GR13-1850). The zeroth-order and first-order diffraction efficiencies are $29.6 \%$ and $53.5 \%$ for the ruled grating, and $78.8 \%$ and $8.3 \%$ for the holographic grating. The scattered noise of the holographic grating is less than the ruled grating, but the difference is not significant. The length of the external cavity is around $110 \mathrm{~mm}$. A beam splitter is inserted in the external cavity, and reflects part of the beam as a diagnostic beam, in which the spectral bandwidth and tunability of the laser system are measured. The zeroth-order diffracted beam of the grating is the output beam of the external-cavity diode laser system. The output power of the diode laser system is measured in this beam. The diode laser is operated at ambient temperature.

\section{RESULTS}

First, the freely running laser device is characterized. The power/current characteristics of the freely running diode laser is measured and shown in Fig. 2. The threshold current of the laser device is around $0.12 \mathrm{~A}$, and the slope efficiency is around $1.62 \mathrm{~W} / \mathrm{A}, 2 \mathrm{~W}$ output power is obtained with an injected current of around 1.4 A. The spectrum of the GaN laser device is measured in freely running condition by a spectrum analyzer (Advantest Corp. Q8347). A typical measured result with an injected current of $0.6 \mathrm{~A}$ is shown in Fig. 3. The diode laser is operating in multiple Fabry-Perot (FP) modes. The optical spectrum is centered at $456 \mathrm{~nm}$, and the spectral bandwidth is around $1.2 \mathrm{~nm}$ (the resolution of the spectrum analyzer is $5 \mathrm{pm}$ ). The free spectral range of the FP laser resonator is around $28.5 \mathrm{pm}$. This corresponds to a cavity length of around $1.5 \mathrm{~mm}$ based on the value of the refractive index of GaN at $455 \mathrm{~nm}$ [18].

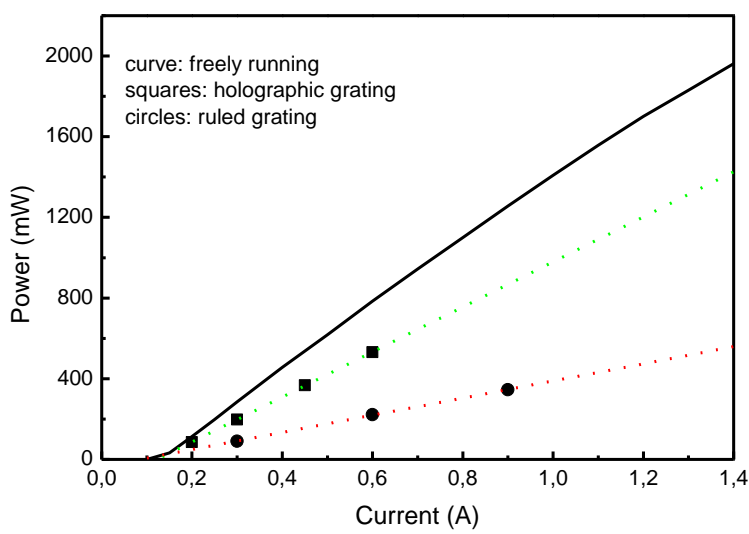

Fig. 2. Optical output power versus injected current for the freely running diode laser (solid curve), external cavity diode laser system with a holographic grating (squares) and a ruled grating (circles). The dotted lines are linear fits to the measured points as a guide to the eye.

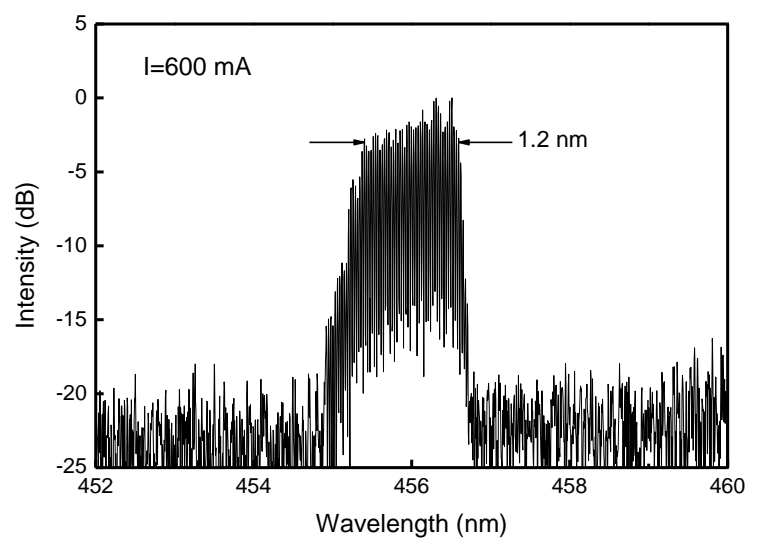

Fig. 3. Optical spectrum of the freely running blue diode laser with an injected current of $0.6 \mathrm{~A}$.

The tunability of the external-cavity diode laser system is characterized by measuring the optical spectrum of the output beam at different wavelengths with the same spectrum analyzer. Fig. 4 shows the measured spectra of the external-cavity diode laser system with output power around $85 \mathrm{~mW}$ with both diffraction gratings. Seven normalized spectra from 453.4 to $456.5 \mathrm{~nm}$ are shown in Fig. 4 (a) for the diode laser system with the holographic diffraction grating at an injected current of $0.2 \mathrm{~A}$. The spectral bandwidth (FWHM) is around 9 $\mathrm{pm}$ in the $3.1 \mathrm{~nm}$ tunable range, much less than the $28.5 \mathrm{pm}$ free spectral 
range of the FP laser resonator, meaning that the laser operates in a single longitudinal FP mode. Fig. 4 (b) shows eight normalized spectra from 452.8 to $458.8 \mathrm{~nm}$ for the external-cavity diode laser system with the ruled diffraction grating at an injected current of $0.3 \mathrm{~A}$. The spectral bandwidth is less than $20 \mathrm{pm}$ for wavelengths longer than $456 \mathrm{~nm}$ in the $6.0 \mathrm{~nm}$ tunable range (Fig. 7 (b)), i.e., single longitudinal mode oscillation occurs. For shorter wavelengths, the spectral bandwidth is less than 42 pm (Fig. 7 (b)), i.e., two longitudinal modes of the FP diode device oscillate simultaneously. The amplified spontaneous emission (ASE) is more than $20 \mathrm{~dB}$ suppressed in the tunable ranges for both diffraction gratings. If we operate the laser system outside of the tunable ranges, the freely running emission around $455.5 \mathrm{~nm}$ appears and dominates the laser output, due to the selected wavelength being far from the gain center of the active medium of the laser device and that no AR coating is applied to the diode laser to suppress lasing under freely running conditions.

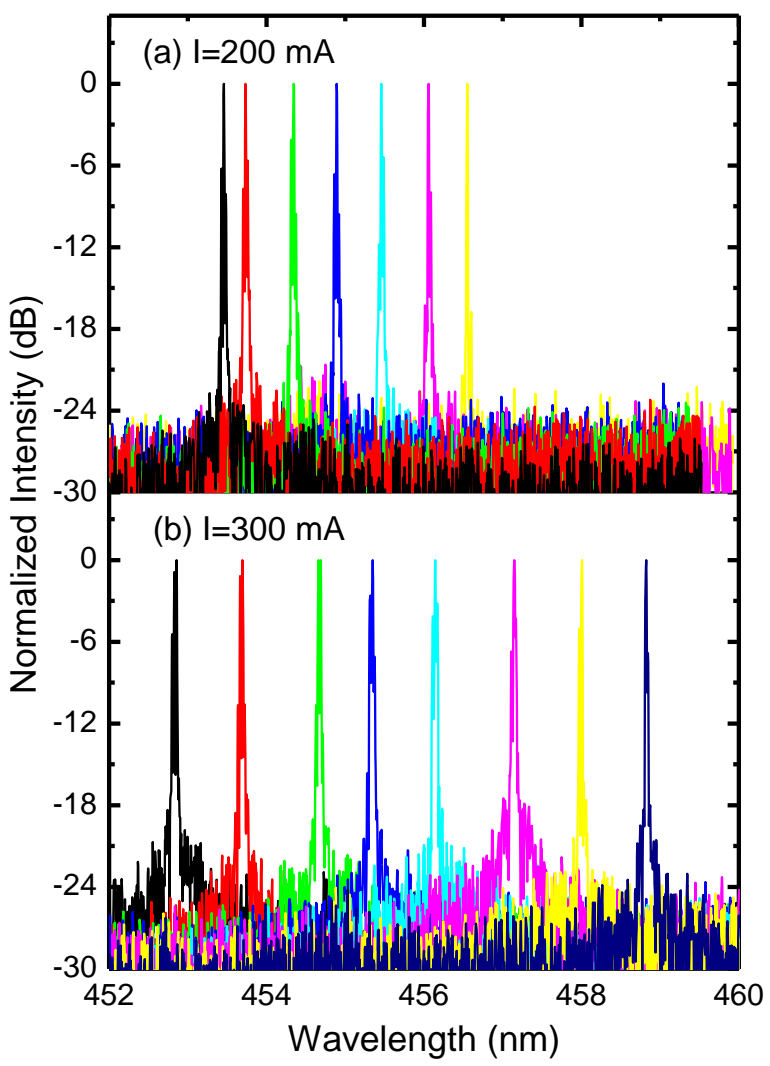

Fig. 4. Optical spectra of the output beam from the external-cavity diode laser with a holographic grating (a) and a ruled grating (b). The output power is around $85 \mathrm{~mW}$.

Figure 5 shows the measured spectra of the external-cavity diode laser system with output power around $200 \mathrm{~mW}$ with both diffraction gratings. Fig.5(a) shows seven normalized spectra of the external-cavity diode laser system from 453.7 to $456.5 \mathrm{~nm}$ with the holographic diffraction grating, at an operating current of $0.3 \mathrm{~A}$. The spectral bandwidth is around $35 \mathrm{pm}$ in the $2.8 \mathrm{~nm}$ tunable range, i.e., two longitudinal modes of the FP diode laser oscillate simultaneously. Fig. 5(b) shows eight normalized spectra of the external-cavity diode laser from 453.5 to $459.0 \mathrm{~nm}$ with the ruled diffraction grating, the injected current is $0.6 \mathrm{~A}$. The spectral bandwidth is less than $67 \mathrm{pm}$ in the $5.5 \mathrm{~nm}$ tunable range, indicating that two or three longitudinal modes of the FP diode laser oscillate simultaneously. Fig. 5(a) shows an ASE suppression of more than $24 \mathrm{~dB}$ in the tunable range; Fig. 5(b) shows suppression of ASE of more than $20 \mathrm{~dB}$ in the tunable range exceptat $459.0 \mathrm{~nm}$ (purple curve in Fig. 5(b)), where the freely running emission weakly appears around $456 \mathrm{~nm}$ and the main peak is around $17 \mathrm{~dB}$ higher than the freely running emission.

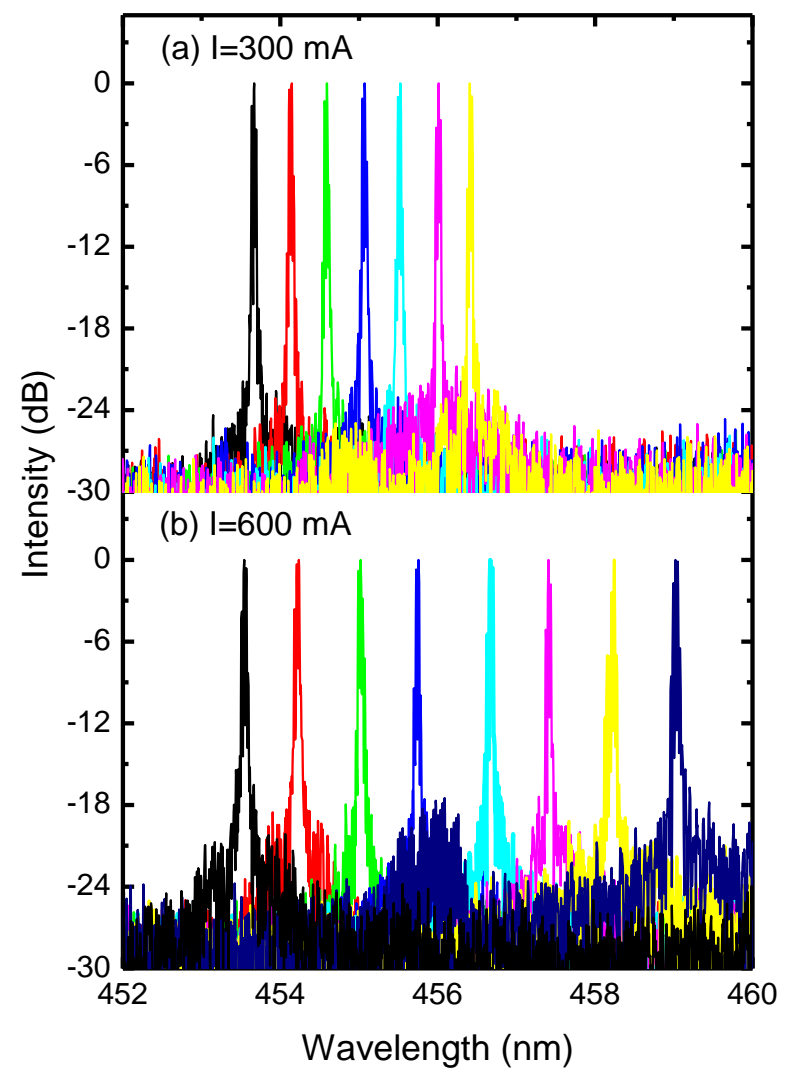

Fig. 5. Optical spectra of the output beam from the external-cavity diode laser with a holographic grating (a) and a ruled grating (b). The output power is around $200 \mathrm{~mW}$.

Figure 6 shows the tunability of the external-cavity diode laser with output power around $350 \mathrm{~mW}$. When the holographic grating is used, the diode laser system is tunable from 454.1 to $456.5 \mathrm{~nm}$ with an injected current of $0.45 \mathrm{~A}$. Here, the spectral bandwidth is around $35 \mathrm{pm}$ in the $2.4 \mathrm{~nm}$ tunable range and the ASE is more than $24 \mathrm{~dB}$ suppressed in the tunable range. When the ruled grating is used, the diode laser is tunable from 455.3 to $459.4 \mathrm{~nm}$ with an injected current of $0.9 \mathrm{~A}$ and the ASE is more than $18 \mathrm{~dB}$ suppressed in the $4.1 \mathrm{~nm}$ tunable range. The spectral bandwidth is less than $70 \mathrm{pm}$ according to the definition of FWHM, but as shown in Fig. 6 (b), side peaks appear in the optical spectrum in some cases.

Using the ruled grating, a further increase of the injected current increases the spectral bandwidth to a few hundred picometres, and two peaks are present in the optical spectrum. With the holographic grating, the operating current can be further increased to $0.6 \mathrm{~A}$, where the diode laser can be tuned from 455.0 to $456.4 \mathrm{~nm}$ with an output power around $530 \mathrm{~mW}$. The spectral bandwidth is around $63 \mathrm{pm}$ in the $1.4 \mathrm{~nm}$ tunable range, and the ASE is more than $20 \mathrm{~dB}$ suppressed. Further increase of the operating current also increases the spectral bandwidth to a few hundred picometres for the external-cavity diode laser with the holographic grating. 
With the two bulk diffraction gratings, the output power of the diode laser system at different wavelengths is measured and the results are shown in Fig. 7(a). With the holographic grating, the maximum output powers are 85, 198, 368 and $531 \mathrm{~mW}$ with injected currents of $0.2,0.3$, 0.45 and $0.6 \mathrm{~A}$, respecively. With the ruled grating, the maximum output powers are 90, 222 and $340 \mathrm{~mW}$ with injected currents of $0.3,0.6$ and 0.9 A, respectively. The output power is relatively constant in the tunable range at each injected current for both diffraction gratings. The figure also shows a redshift of the position of the peak power with an increase of the injected current. The reason is the redshift of the gain center of the gain medium with the increasing inject current and increased thermal load [11]. We also note that the redshift is more pronounced when using the ruled grating as the operating current and thus the thermal load is higher at comparable output power.

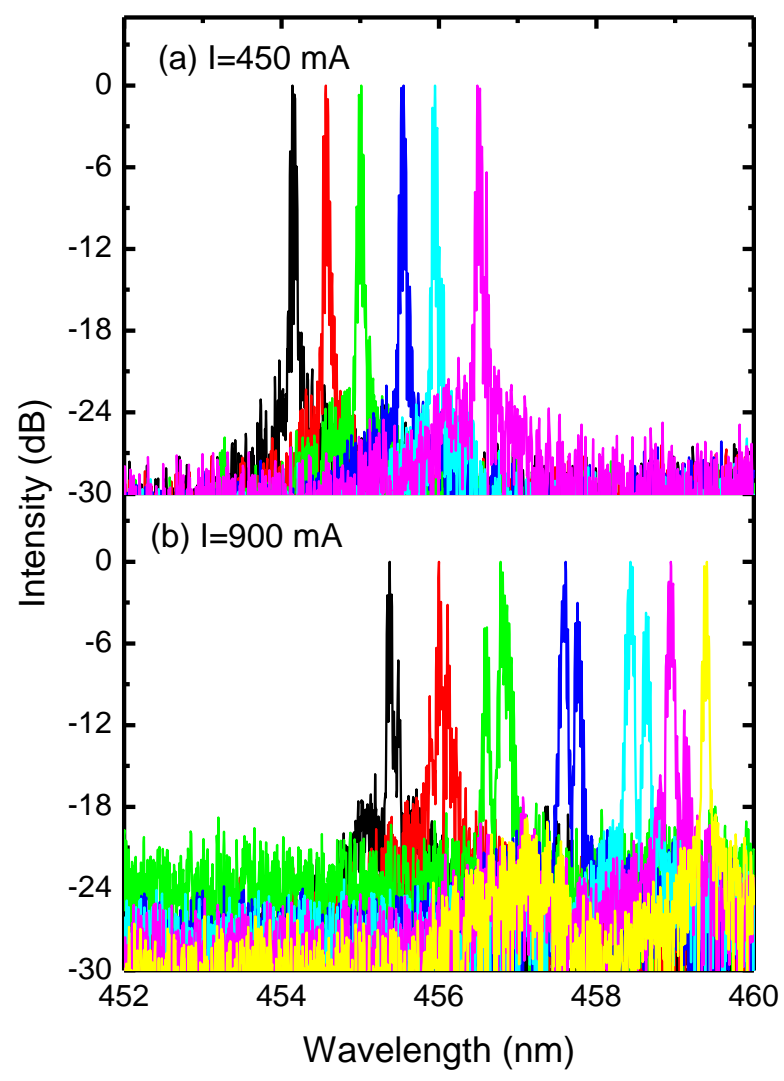

Fig. 6. Optical spectra of the output beam from the external-cavity diode laser with a holographic grating (a) at an injected current of $0.45 \mathrm{~A}$, and a ruled grating (b) at an injected current of $0.9 \mathrm{~A}$.

In order to compare the efficiency of the external-cavity diode laser system with different diffraction gratings, the maximum powers ateach injected current for both diffraction gratings are included in Fig. 2 . The efficiency of the diode laser system with the holographic grating is much higher than that of the laser system using the ruled grating. At the maximum output power with both gratings, i.e., $531 \mathrm{~mW}$ at the injected current of $0.6 \mathrm{~A}$ for the holographic grating and $340 \mathrm{~mW}$ at the injected current of $0.9 \mathrm{~A}$ for the ruled grating, $67.7 \%$ and $27.1 \%$ of the output power of the freely running laser diode is extracted in the externalcavity diode laser system for the two gratings individually. This includes the additional loss of the inserted beam splitter. The main reason for this difference is the higher diffraction efficiency of the ruled grating limiting the output power.
The spectral bandwidth at each wavelength and injected current is shown in Fig. 7 (b) for both diffraction gratings. When the output power is comparable for both diffraction gratings, the spectral bandwidth obtained with the holographic grating is narrower than that obtained with the ruled grating, especially when the output power is low. The spectral bandwidth is less than $70 \mathrm{pm}$ throughout the tunable ranges at all injected currents for both diffraction gratings.

Here we should mention that the diode laser applied in our experiment is a broad-area, multi-lateral mode diode laser. A far-field profile with multi peaks is observed, and the profile is almost unchanged by the external-cavity feedback.

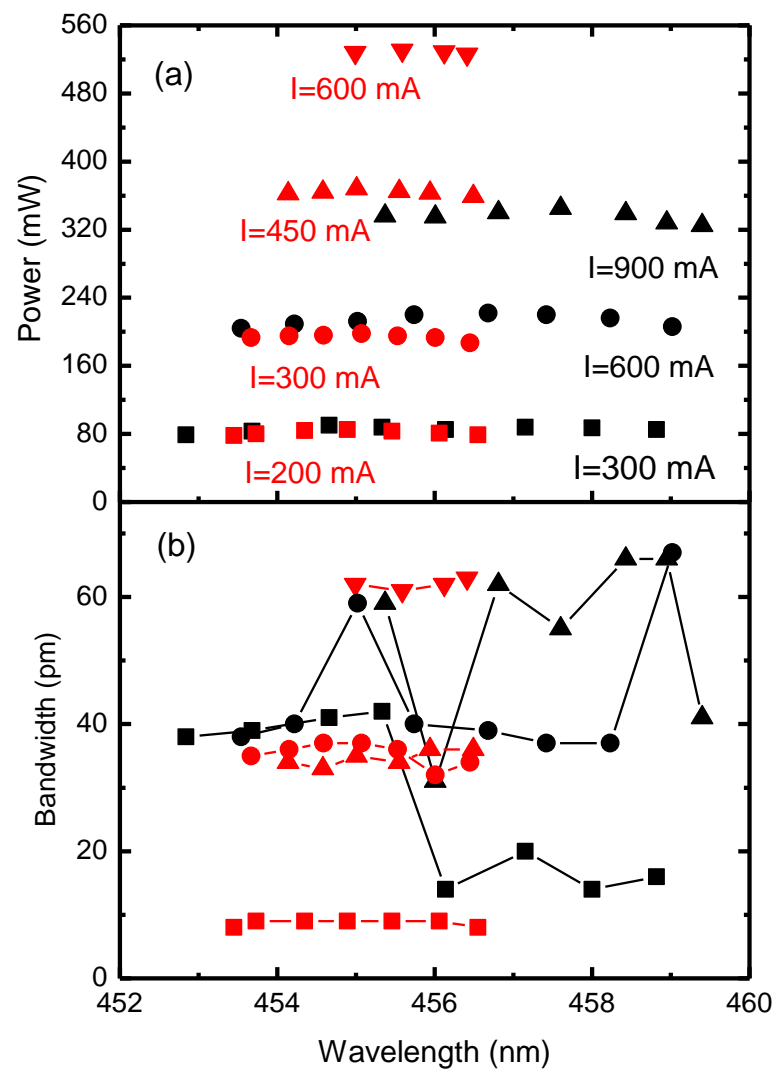

Fig. 7. Output power (a) and spectral bandwidth (b) of the externalcavity diode laser at different wavelengths and operating currents, with a holographic grating (red signs) and a ruled grating (black signs).

\section{DISCUSSION}

Table 1 shows the tunable range and output power of external-cavity GaN diode laser published previously, and the results of this paper. In the present work, with an injected current of $0.3 \mathrm{~A}$, a $6 \mathrm{~nm}$ tunable range is obtained with an output power around $85 \mathrm{~mW}$ when the ruled grating is applied in the external-cavity diode laser. This tunable range is comparable with a $6.3 \mathrm{~nm}$ tuning range achieved with an antireflection-coated InGaN laser diode [10], but the output power of the latter laser system was only a few $\mathrm{mW}$. Using an antireflectioncoated GaN laser diode, Hildebrandt et al. achieved an external-cavity diode laser with a tunable range of $4 \mathrm{~nm}$ and an output power of $30 \mathrm{~mW}$ [1]. With a holographic grating, a $400 \mathrm{~mW}$ output power was obtained from an external-cavity diode laser system around $445 \mathrm{~nm}$ [13]. When the holographic grating is used, a $530 \mathrm{~mW}$ output power is achieved in the present laser system. We believe a wider tunable range and higher 
output power can be obtained if an antireflection coating is applied to our diode laser.

Table 1. Summary of the Main Parameters for the External-cavity Diode lasers in This Paper and Previous Publications

\begin{tabular}{cccc}
\hline & \multicolumn{3}{c}{ lasers in This Paper and Previous Publications } \\
\cline { 2 - 4 } $\begin{array}{c}\text { Laser } \\
\text { system }\end{array}$ & $\begin{array}{c}\text { Wavelength } \\
\text { (nm) }\end{array}$ & Tunable range & $\begin{array}{c}\text { Output } \\
\text { power (mW) }\end{array}$ \\
\hline Ref. [1] & 405 & $4.0 \mathrm{~nm}$ & 30 \\
\hline Ref. [9] & 392 & $2.7 \mathrm{~nm}$ & 3.5 \\
\hline Ref. [10] & 398 & $6.3 \mathrm{~nm}$ & 5.0 \\
\hline Ref. [11] & 450 & $110 \mathrm{GHz}$ (mode-hop- & 0.9 \\
& & free) & \\
\hline Ref. [12] & 405 & $4.5 \mathrm{~nm}$ & 1.0 \\
\hline Ref. [13] & 445 & $3.0 \mathrm{~nm}$ & 400 \\
\hline $\begin{array}{c}\text { Present } \\
\text { work }\end{array}$ & 455 & $6.0 \mathrm{~nm}$ & 80 \\
& & $1.4 \mathrm{~nm}$ & 530 \\
\hline
\end{tabular}

Comparing the results obtained with the two diffraction gratings, the external-cavity diode laser system with the holographic grating has narrower spectral bandwidth and larger suppression of ASE when the output power of the diode laser system with the two gratings are comparable. A possible reason is that the holographic grating has a larger groove density compared with the ruled grating, i.e., 2400 lines/mm for the holographic grating vs 1800 lines/mm for the ruled grating, meaning that the holographic grating has a higher spectral resolution. Furthermore, the laser is operated at lower currents using the holographic grating further suppressing the freely running operation of the diode laser.

The efficiency of the diode laser system with the holographic grating is much higher than that of the diode laser system with the ruled grating, due to the fact that the holographic grating has a much higher zerothorder diffraction efficiency compared with the ruled grating, i.e., $78.8 \%$ for the holographic grating vs $29.6 \%$ for the ruled grating. The tunable range of the diode laser system with the ruled grating is around two times that of the diode laser system with the holographic grating when the output power of the diode laser system is comparable. The reason is that the ruled grating has a much higher first-order diffraction efficiency (53.5\%) compared with the holographic grating (8.3\%), and the higher the feedback strength from the grating, the more effective the suppression of the freely running lasing modes. Thus there is a compromise between the output power and tunable range of the external-cavity diode laser since the sum of the zeroth-order and firstorder diffraction efficiencies is around unity.

To understand the compromise between the tunable range and output power further, theoretical analysis based on rate equation of diode laser is needed. The rate equations describing the carrier density $N$ and photon density $N_{\text {ph }}$ in the diode laser cavity are given as [1,19]:

$$
\begin{aligned}
& \frac{d N}{d t}=\frac{\eta_{i} j}{q d}-\frac{N}{\tau}-v_{g r} g(N) N_{p h}, \\
& \frac{d N_{p h}}{d t}=v_{g r} \Gamma g(N) N_{p h}-\frac{N_{p h}}{\tau_{p h}}+\alpha_{m} v_{g r} K N_{p h}\left(t-t_{0}\right),
\end{aligned}
$$

where $j$ is the inject current density, $\eta$ is the internal efficiency, $q$ is the elementary charge of an electron, $d$ is the thickness of the active region, $\tau$ is the carrier lifetime, $v_{g r}$ is the group velocity of the photons, $g(N)$ is the material gain, $\Gamma$ is the confinement factor, $\tau_{\mathrm{ph}}$ is the photon lifetime, $\alpha_{\mathrm{m}}$ is the mirror loss, $K$ is the feedback strength of the grating (the firstorder diffraction efficiency in our case, here the other loss in the cavity is neglected.), to is the time delay of the laser lightrunning in the external cavity. In particular $1 / \tau_{\mathrm{ph}}=v_{g r}\left(\alpha_{\mathrm{i}}+\alpha_{\mathrm{m}}\right)$, where $\alpha_{\mathrm{i}}$ is the internal loss; and the gain $g(N)=g_{\mathrm{N}}\left(N-N_{\mathrm{r}}\right), g_{\mathrm{N}}$ is the differential gain coefficient and
$N_{\text {tr }}$ is the transparency carrier density. When the diode laser system is steady-state operated, rearrange the Eq. (1) and (2), we obtain:

$$
\begin{aligned}
& \frac{d N}{d t}=\frac{\eta_{i} j}{q d}-\frac{N}{\tau}-v_{g r} g_{N}\left(N-N_{t r}\right) N_{p h}, \\
& \frac{d N_{p h}}{d t}=v_{g r} \Gamma g_{N}\left(N-N_{t r}\right) N_{p h}-v_{g r}\left[\alpha_{i}+(1-K) \alpha_{m}\right] N_{p h} .
\end{aligned}
$$

Eq. (4) shows the effect of the feedback is to reduce the mirror loss from $\alpha_{\mathrm{m}}$ to $(1-K) \alpha_{\mathrm{m}}$.

The steady-state solution of Eq. (3) and (4) can be obtained as:

$$
\begin{aligned}
& N=N_{t r}+\frac{1}{\Gamma g_{N} \tau_{p h} v_{g r}}-\frac{K \alpha_{m}}{\Gamma g_{N}}=N_{t h}(0)+\frac{K \alpha_{m}}{\Gamma g_{N}}, \\
& N_{p h}=\frac{\eta_{i} \Gamma \tau_{p h}}{q d}\left[j-j_{t h}(K)\right] \frac{1}{1-K \alpha_{m} /\left(\alpha_{i}+\alpha_{m}\right)^{\prime}}, \\
& j_{t h}(K)=j_{t h}(0)-\frac{q d K \alpha_{m}}{\eta_{i} \tau \Gamma g_{N}},
\end{aligned}
$$

where $N_{\mathrm{th}}(0)$ is the threshold carrier density without feedback, and $j_{t h}(0)=q d N_{t h}(0) / \eta_{i} \tau$ is the threshold current density without feedback. Eq. (6) shows the threshold carrier density $j_{t h}(K)$ is decreased with the feedback strength $K$ The photon density increases with the feedback strength $K$.
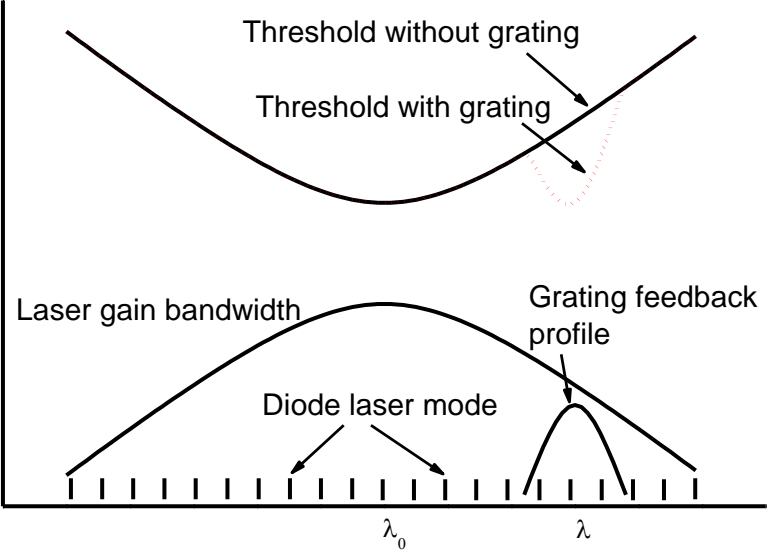

Fig. 8. Schematic diagram of the gain and threshold with and without grating feedback. The grating feedback profile is also indicated.

In our experiment, when we rotate the grating further to operate the laser system outside of the tunable ranges, the freely running emission appears and dominates the laser output, this means the competition of the freely running lasing at the gain center $\lambda_{0}$ and the lasing at wavelength $\lambda$ (with a distance from the gain center) with feedback determines the tunable range of the external cavity diode laser system. Fig. 8 shows a schematic diagram of the gain and the threshold of the diode laser system, the grating feedback profile is also shown. The grating feedback decreases the threshold current density at $\lambda$ from $j_{t h}(\lambda, 0)$ to $j_{t h}(\lambda, K)$. When $j_{t h}(\lambda, K)$ is less than the freely running threshold current density at $\lambda_{0}$, i.e., $j_{t h}\left(\lambda_{0}, 0\right)$, the laser system lases at $\lambda$, and vise versa. Since the decrease of the threshold current density by the feedback is proportional to $K$, a higher value of $\mathrm{K}$ cause a wider tunable range of the external-cavity diode laser system.

The output power $P$ of an external-cavity diode laser system can be expressed as:

$$
P \propto N_{p h}(1-K)=\frac{\eta_{i} \Gamma \tau_{p h}}{q d}\left[j-j_{t h}(K)\right] \frac{1-K}{1-K \alpha_{m} /\left(\alpha_{i}+\alpha_{m}\right)} .
$$

The slope efficiency of the laser system decreases with the feedback strength $K$ due to the last term in Eq. (8). Thus the output power decreases with $K$, when the laser system is operated far above the 
threshold; although the threshold of the laser system also decreases with the feedback strength $K$

This above analysis is consistent with our experimental observation, where the laser system with the ruled grating has a much wider tunable range but less output power compared with the laser system with the holographic grating. In Ref. [9], an output power of $3.5 \mathrm{~mW}$ with a tunable range of $1.3 \mathrm{~nm}$ was achieved with a grating of $30 \%$ first-order diffraction efficiency; an output power of $1.5 \mathrm{~mW}$ with a tunable range of $2.7 \mathrm{~nm}$ was achieved with a grating of 55\% first-order diffraction efficiency. In Ref. [10], a $6.3 \mathrm{~nm}$ tunable range was obtained when the first-order diffraction efficiency of the grating is $76 \%$; and a $4.8 \mathrm{~nm}$ tunable range was obtained when the first-order diffraction efficiency of the grating is $46 \%$. All these experimental results support the theoretical analysis above. From the above analysis, we can conclude that the compromise between the output power and tunable range of the external-cavity diode laser is a general condition, and irrelevant to the geometry of the external-cavity.

The experimental results obtained in this paper and previously and the theoretical analysis provide a general guide on selection of grating for external-cavity diode laser systems. Two main parameters of a diffraction grating have to be considered when a grating is chosen to construct an external-cavity diode laser: the groove density and the first-order diffraction efficiency. A larger groove density grating will give a narrower spectral bandwidth and a higher suppression of ASE compared with a grating with small groove density. If a wider tunable range of a laser system is the high priority, a grating with higher firstorder diffraction efficiency should be selected. If a higher output power of a laser system is prioritized, a grating with low first-order diffraction efficiency should be selected. Thus there is a compromise between the tuning range and the output power of an external-cavity diode laser system, and this is the main consideration for selecting a diffraction grating to build an external-cavity diode laser system.

\section{CONCLUSION}

Based on a $455 \mathrm{~nm}$ GaN diode laser and external-cavity grating feedback, a tunable, narrow-bandwidth, high-power diode laser system is achieved with a holographic grating and a ruled grating. The output power, efficiency, spectral bandwidth and tunable range of the external cavity diode laser system are measured and compared using the two different diffraction gratings at different injected currents. The diode laser system can be tuned over a range of $1.4 \mathrm{~nm}$ with an output power around $530 \mathrm{~mW}$, when the holographic grating is used. An $80 \mathrm{~mW}$ output power in a tunable range of $6 \mathrm{~nm}$ is obtained from the diode laser system with the ruled grating. The comparison of the results obtained with these two gratings provides a guide for selecting gratings for external-cavity diode laser systems for different requirements. The output power and tuning range can be improved further if an antireflection coating is applied to the front facet of the GaN diode laser.

\section{References}

1. L. Hildebrandt, R. Knispel, S. Stry, J. R. Sacher, and F. Achael, “Antireflctioncoated blue GaN laser diodes in an external cavity and Doppler-free indium absorption spectroscopy," Appl. Opt. 42, 2110-2118 (2003).

2. K. Holc, Z. Bielecki, J. Wojtas, P. Perlin, J. Goss, A. Czyzewski, P. Magryta, and T. Stacewicz, "Blue laser diodes for trace matter detection," Opt. Appl. 40, 641-651 (2010).

3. T. Tanaka, K. Takahashi, K. Sako, R. Kasegawa, M. Toishi, K. Watanabe, D. Samuels, and M. Takeya, "Littrow-type external-cavity blue laser for holographic data storage," Appl. Opt. 46, 3583-3592 (2007).

4. Y. Shimada, Y. Chida, N. Ohtsubo, T. Aoki, M. Takeuchi, T. Kuga, and Y. Torii, “A simplified 461-nm laser system using blue diodes and a hollow cathode lamp for laser cooling of Sr," Rev. Sci. Instrum. 48, 063101 (2013).

5. N. Ruhnke, A. Müller, B. Eppich, R. Güther, M. Maiwald, B. Sumpf, G. Erbert, and G. Tränkle, "Single-pass UV generation at $222.5 \mathrm{~nm}$ based on high-power GaN external cavity diode laser," Opt. Lett. 40, 2127-2129 (2015).

6. S. Nakamura, M. Senoh, S. Nagahama, N. Iwasa, T. Yamada, T. Matsushita, Y. Sugimoto, and H. Kiyoku, "Room-temperature continuouswave operation of InGaN multi-quantum-well structure laser diodes," Appl. Phys. Lett. 69, 4056-4058 (1996).

7. http://www.osram-os.com

8. http://www.nichia.co.jp

9. R. S. Conroy, J. J. Hewett, G. P. T. Lancaster, W. Sibbett, J. W. Allen, and K. Dholakia, "Characterisation of an extended cavity violet diode laser," Opt. Commun. 175, 185-188 (2000).

10. D. J. Lonsdale, A. P. Willis, and T. A. King, "Extended tuning and singlemode operation of an anti-reflection-coated InGaN violet laser diode in a Littrow cavity," Meas. Sci. Technol. 13, 488-493 (2002).

11. J. Hult, I. S. Burns, and C. F. Kaminski, "Wide-bandwidth mode-hop-free tuning of extended-caavity GaN diode lasers," Appl. Opt. 44, 3675-3685 (2005).

12. X. Lv, S. Chen, J. Zhang, L. Ying, B. Zhang, "Tuning properties of external cavity violet semiconductor laser," Chin. Phys. Lett. 30, 074204 (2013).

13. N. Ruhnke, A. Müller, B. Eppich, M. Maiwald, B. Sumpf, G. Erbert, and G. Tränkle, " $400 \mathrm{~mW}$ external cavity diode laser with narrowband emission at 445 nm," Opt. Lett. 39, 3794-3797 (2014).

14. M. G. Littman and H. J. Metcalf, "Spectrally narrow pulsed dye laser without beam expander," Appl. Opt. 17, 2224-2227 (1978).

15. J. F. Sell, W. Miller, D. Wright, B. V. Zhdanov, and R. J. Knize, "Frequency narrowing of a $25 \mathrm{~W}$ broad area diode laser," Appl. Phys. Lett. 94, 051115 (2009).

16. M. Chi, G. Erbert, B. Sumpf, and P. M. Petersen, "Tunable high-power narrow-spectrum external-cavity diode laser based on tapered amplifier at $668 \mathrm{~nm}$," Opt. Lett. 35, 1545-1547 (2010).

17. M. Chi, O. B. Jensen, and P. M. Petersen, "High-power dual-wavelength external-cavity diode based on tapered amplifier with tunable terahertz frequency difference," Opt. Lett. 36, 2626-2628 (2011).

18. U. Tisch, B. Meyler, O. Katz, E. Finkman, and J. Salzman, "Dependence of the refractive index of AlxGa1-xN on temperature and composition at elevated temperatures," J. App. Phys. 89, 2676-2685 (2001).

19. P. Unger, "Introduction to power diode laser," in High-power diode lasers, Fundamentals, Technology, Applications, R. Diehl, ed. (Springer-Verlag, Berlin Heidelberg, 2000), pp. 37-46. 